\title{
TRANSMISSION OF IMAGE USING SMS TECHNIQUE
}

\author{
Ashish A. Mahajan', Y. D. Chincholkar ${ }^{2}$ \\ ${ }^{I}$ Dept. of Electronics and Telecommunications, Sinhgad College of Engineering, Pune, India \\ ${ }^{2}$ Dept. of Electronics and Telecommunications, Sinhgad College of Engineering, Pune, India
}

\begin{abstract}
Communication is very important ingredient of life which involves transfer of photos and images through mail or other applications using internet connection to effectively explain the situation. But if the internet connection is not there, can one send photos and images to each other. Thus, this paper is an attempt to provide a solution for the ones who regularly use SMS for communication and have to show any image or reference about the picture to another person, who is far away, without the internet connection. This technique will be helpful to the people using any mobile phone as efficiently and effectively as possible and achieve its goal of image transfer without the internet connection. The basic idea involves image processing, color image conversion and compression and then transferring image from one device to another using the SMS technique.
\end{abstract}

Keywords: Image Processing, Image conversion, Image compression, SMS Technique.

$* * *$

\section{INTRODUCTION}

Now-a-days, everyone is using SMS in a tremendous way for communicating with each other as mobile communication has become one of the fastest means of communication. SMS is an integral part of data communication between the mobile phones. But sometimes people need to share images for giving the reference of the topic on which the discussion is going on. This image sharing can be done through use of internet but what if there is no internet connection and still the image needs to be shared. Thus, in that case, the SMS Technique can be used to send the image.

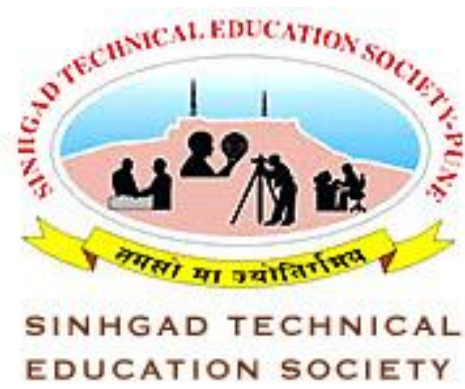

Fig 1 A simple image (example) [12]

Image processing has become the most important field of research due to its variety of applications like image restoration, segmentation, medical image processing and satellite image processing [1]. Basically, images can be twodimensional or three-dimensional, such as photograph, screen display etc. They may be captured by optical devices such as camera etc.

SMS is nothing but a synonym for all the different types of short text messaging. The Global System for Mobile Communications (GSM) series of standards included SMS as its part in 1985. SMS uses standardized communications protocols. It is a text messaging service component of mobile communication systems, phone or web. It allows the exchange of short text messages between fixed line or mobile phone devices [8]. SMS has national and international roaming facilities.

The GSM Architecture provides few other messaging services or technologies like EMS and MMS. They have richer contents than SMS. EMS, Extended Messaging Service, is generally used to send predefined sounds, images and animations but is not widely supported. MMS, Multimedia Messaging Service, is used to transmit graphics, video clips, sound files and short text messages using a storeand-forward method using the WAP protocol over wireless networks like GPRS, 3G which demands up gradation in the network. SMS text messaging is tremendously used all over the world making it the most widely used data application with over 3.7 billion active users , or $74 \%$ of all mobile phone subscribers.

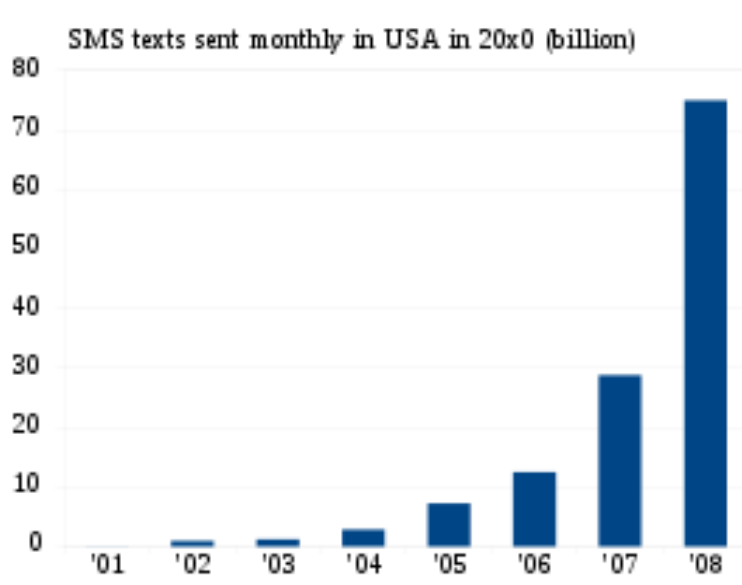

Fig 2 SMS messages sent monthly in USA (billion) [8]

SMS is a medium used for sending messages having length of 160 characters, to and from GSM mobile handsets. It is very important that the length of single message is restricted to 140 bytes, which is 160 seven-bit characters; so that the existing signaling formats can support the messages [11]. The 
text message should contain only those characters which are present in the GSM 7-bit character set, so that 140 bytes (8bits) are obtained from 160 seven-bit characters to generate the 160 character limit. The segmentation and reassembly standards are defined, if a message is larger than 140 bytes (8-bits), so that multiple physical SMS messages can be generated and sent over the air for a single logical message. The receiving client should reassemble the segmented message so that the receiving device can view it as a single message.

\section{LITERATURE SURVEY}

The Literature Survey involves the study of RGB Color Model, Color Depth and the different types of images, namely color image, grayscale image and binary image that can be used to transfer from one device to another. The RGB color model is nothing but an additive color model. A broad array of colors can be obtained by adding together red, green, and blue light in various ways. The RGB color model is mainly used for display of images, sensing the images and representation the images in electronic systems which includes phones, televisions and computers.

A single pixel consists of various numbers of bits known as color depth or bit depth which is used to indicate the color in a bitmapped image quantified as bits per pixel (bpp). The grayscale digital image is an image in which is composed exclusively of shades of gray. It varies from weakest intensity to strongest intensity which means from black to white. Each pixel has a value of single sample and it carries only intensity information [1]. Now-a-days, grayscale images are commonly stored with 8 bits per sampled pixel intended for visual display. This helps to obtain 256 different intensities (i.e., shades of gray).

It also involves the study of existing techniques or to check what all techniques are available to transfer an image from one device to another like MMS Technique, Bluetooth Technique, OTA Bitmap Technique by Nokia, Attaching through Mail, through some android applications like WhatsApp etc. MMS makes the use of carrier's MMSC store and forward server which is known as MMSC for delivering the messages which is completely different way from SMS. Bluetooth uses short-wavelength radio transmissions for exchanging data over short distances. It is a wireless technology standard. It cannot be used to transfer the data for long distance. OTA Bitmap or Over The Air Bitmap was designed for black and white images by Nokia to send pictures as a series of one or more concatenated SMS text messages [9].

\section{RELATED WORK}

C. Saravanan has proposed a new algorithm to convert the color image into grayscale image and preserve sharpness contrasts, shadow, and structure of the color image [1]. Sherif A. Mohamed and Moustafa M. Fahmy has proposed a new binary image coding technique in which firstly the black regions in the input image are partitioned into a number of nonoverlapping rectangles and then each rectangle consists of two opposite vertices. The coordinates of these two opposite vertices are compressed using a simple procedure that allows the decoder to reconstruct the original image [2]. Pasi Fränti and Olli Nevalainen has proposed a new two-stage modelling schema that is used along with arithmetic coding which helps to consume less time [3]. M. Kafashan, H.Hosseini, S.Beygiharchegani, P.Pad and F.Marvasti have proposed two lossless compression techniques that represent a two dimensional Run-length coding which can achieve high compression ratio [4]. Bekir Karlık and Mustafa Sarıöz have presented a novel method on coloring the grayscale images by combination of artificial neural networks and some image processing algorithms was developed using which the colors from a user-selected source image can be transferred to a target grayscale image [5]. Mrs. Smriti Kumar and Mr. Ayush Swarnkar have introduced a general technique for "colorizing" grayscale images by transferring color from a source color image to a destination or target grayscale image [6]. Melander, Per (SE) has introduced a method of processing data to be transferred in an SMS message in accordance with a predetermined conversion table [7].

\section{PROPOSED WORK}

The proposed method is that the image will be converted into characters and transmitted through SMS technique by the sender and the receiver will convert the characters back to image. The block diagram of "Transmission of image using SMS Technique" is as shown:

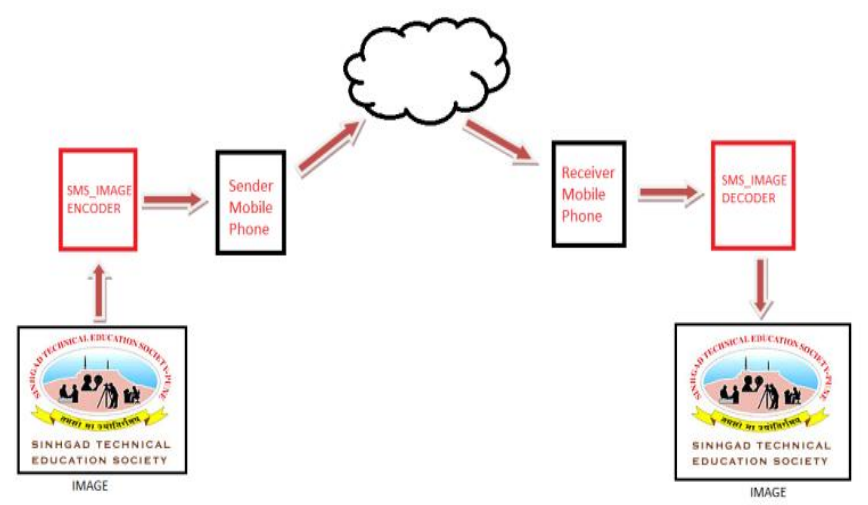

Fig 3 Proposed Block Diagram of Transmission of image using SMS Technique

The Elements involved in the Block Diagram are Sending Image, SMS_IMAGE Encoder, Sender Mobile Phone, Transmission Cloud, Receiver Mobile Phone, SMS_IMAGE Decoder, and Received Image. This method will help all the mobile phones to transfer the image from one mobile to another even if they are very far and without internet connection. The images can be of different sizes. Hence as the size of the image increases, the number of sections increases which will ultimately increase the number of messages sent between sender and receiver. As the image size increases, the complexity also increases. Compression and De-compression techniques can be used for reducing the number of messages required for sending the image. 


\section{METHODOLOGY}

The main function of SMS_IMAGE ENCODER is to convert the IMAGE into an SMS and the main function of SMS_IMAGE DECODER is to convert the SMS into an IMAGE. The conversion of color image into grayscale image can be done using the algorithm proposed by C. Saravanan [1] which performs RGB approximation, reduction and addition of luminance and chrominance. The compression can be performed using the new rectangular partitioning lossless compression technique [4]. It involves the transmission of length and width of each rectangle instead of the location of the vertices. It is as shown below:

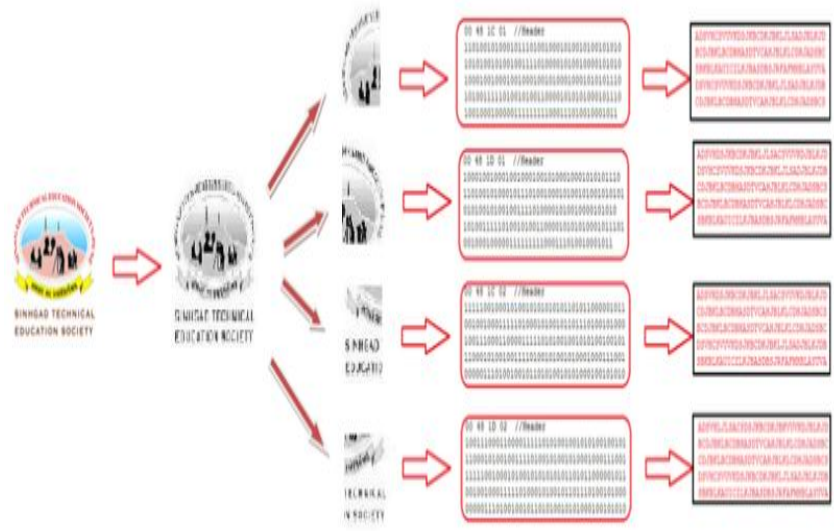

Fig 4 SMS_IMAGE ENCODER Process Diagram

The Steps involved in SMS_IMAGE ENCODER are:

Convert the color image into grayscale image, Divide the Image into smaller sections, Each Section is converted into its Binary form along with the header information containing that sections position in the original image, Convert the Binary Data of each section into the SMS Characters form of length maximum 160 characters.

The main function of SMS_IMAGE DECODER is to convert the SMS into an IMAGE. It is as shown below:

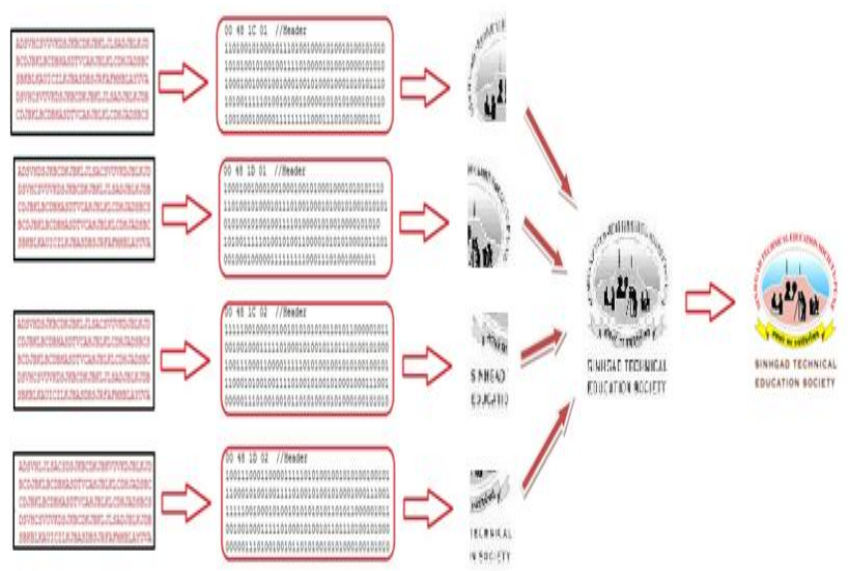

Fig 5 SMS_IMAGE DECODER Process Diagram

The Steps involved in SMS_IMAGE DECODER are: Convert the received SMS of length maximum 160 Characters into Binary Data, Depending on the header information, determine that sections position in the original image and convert the characters into binary form, Arrange the received sections according to the header information to regain the grayscale image, Convert the grayscale image to original image.

\section{RESULTS}

The proposed methodology is tested using the Sinhgad college logo image and Orange-sun-small. The Android SDK tool software has been used for testing. The result mainly focuses on the number of characters generated from the color image and the grayscale image and the total number of SMS's required for transmitting it without involving any compression technique.

Table 1 Experimental results of image conversion without any compression.

\begin{tabular}{|l|l|l|}
\hline Image Name & Sinhgad college logo & $\begin{array}{l}\text { Sinhgad college } \\
\text { logo }\end{array}$ \\
\hline $\begin{array}{l}\text { Conversion } \\
\text { method }\end{array}$ & Color image -> Text & $\begin{array}{l}\text { Color image -> } \\
\text { Grayscale image - } \\
>\text { Text }\end{array}$ \\
\hline $\begin{array}{l}\text { No. of } \\
\text { characters } \\
\text { generated }\end{array}$ & 25387 & 19366 \\
\hline $\begin{array}{l}\text { SMS Header } \\
\text { length characters }\end{array}$ & 10 characters & $130 \mathrm{sms}$ \\
\hline $\begin{array}{l}\text { Total number of } \\
\text { SMS's }\end{array}$ & $170 \mathrm{sms}$ & \\
\hline
\end{tabular}

\begin{tabular}{|l|l|l|}
\hline Image Name & Orange-sun-small & Orange-sun-small \\
\hline $\begin{array}{l}\text { Conversion } \\
\text { method }\end{array}$ & Color image -> Text & $\begin{array}{l}\text { Color image -> } \\
\text { Grayscale image - } \\
>\text { Text }\end{array}$ \\
\hline $\begin{array}{l}\text { No. of } \\
\text { characters } \\
\text { generated }\end{array}$ & 1727 & 1352 \\
\hline $\begin{array}{l}\text { SMS Header } \\
\text { length }\end{array}$ & 10 characters & 10 characters \\
\hline $\begin{array}{l}\text { Total number of } \\
\text { SMS's }\end{array}$ & 12 sms & 10 sms \\
\hline
\end{tabular}

As seen from the results, the number of characters generated by color image is more than the number of characters generated by grayscale image. Hence, converting grayscale image into text requires less number of SMS's for sending as compared to color image.

\section{CONCLUSIONS}

The "Transmission of image using SMS Technique" helps to transfer the image from one mobile phone to another using the SMS technique. It provides a solution for the people who regularly use SMS for communication and want to use any image or reference about the picture to explain their view or points to another person, who is far away, without the use of internet connection. 


\section{REFERENCES}

[1]. C. Saravanan, "Color Image to Grayscale Image Conversion", 2010, International Conference on Computer Engineering and Applications, Pages 196-199.

[2]. Sherif A. Mohamed and Moustafa M. Fahmy, "Binary image compression using efficient partitioning into rectangular regions" IEEE Trans. on Comm., vol. 43, No. 5, pp. 1888-1893, 1995.

[3]. Pasi, F. and Olli, N., 1993. "Two-stage modelling method for compressing binary images by arithmetic coding". Computer Journal 36, pp. 615-622.

[4]. M. Kafashan, H.Hosseini, S.Beygiharchegani, P.Pad and F.Marvasti, New Rectangular Partitioning Methods for Lossless Binary Image Compression, 2010, ACRI.

[5]. Bekir Karlık and Mustafa Sarı̈z, "Coloring Gray-Scale Image Using Artificial Neural Networks", 2009, IEEE. [6]. Mrs. Smriti Kumar, Mr. Ayush Swarnkar, "Colorization of Gray Scale Images in $1 \alpha \beta$ Color Space Using Mean and Standard Deviation", 2012, IEEE.

[7]. European Patent Application EP1555840: Converting binary data into SMS characters.

[8]. http://en.wikipedia.org/wiki/Short_Message_Service

[9]. http://en.wikipedia.org/wiki/OTA_bitmap

[10]. http://en.wikipedia.org/wiki/Grayscale

[11]. LA Times: Why text messages are limited to 160 characters

[12]. http://www.sinhgad.edu/sinhgad-engineering-institutes/ 